\title{
A conserved family of calcineurin regulators
}

\author{
Tami J. Kingsbury and Kyle W. Cunningham ${ }^{1}$ \\ Department of Biology, Johns Hopkins University, Baltimore, Maryland 21218 USA
}

The protein phosphatase calcineurin mediates many cellular responses to calcium signals. Using a genetic screen in yeast, we identified a new family of proteins conserved in fungi and animals that inhibit calcineurin function when overexpressed. Overexpression of the yeast protein Ren1p or the human homologs DSCR1 or ZAKI-4 inhibited two independent functions of calcineurin in yeast: The activation of the transcription factor Tcn1p and the inhibition of the $\mathrm{H}^{+} / \mathrm{Ca}^{2+}$ exchanger Vex1p. Purified recombinant Rcn1p and DSCR1 bound calcineurin in vitro and inhibited its protein phosphatase activity. Signaling via calmodulin, calcineurin, and Tcn1p induced Ren1p expression, suggesting that Ren1p operates as an endogenous feedback inhibitor of calcineurin. Surprisingly, rcn1 null mutants exhibited phenotypes similar to those of Rcn1p-overexpressing cells. This effect may be due to lower expression of calcineurin in rcn1 mutants during signaling conditions. Thus, Rcn1p levels may fine-tune calcineurin signaling in yeast. The structural and functional conservation between Rcn1p and DSCR1 suggests that the mammalian Rcn1p-related proteins, termed calcipressins, will modulate calcineurin signaling in humans and potentially contribute to disorders such as Down Syndrome.

[Key Words: Calcineurin; calcium signaling; Rcn1p; DSCR1; ZAKI-4]

Received February 21, 2000; revised version accepted May 18, 2000.

The calcium and calmodulin-activated protein phosphatase calcineurin regulates a variety of developmental and cellular processes. Calcineurin helps control T-cell activation (Liu et al. 1992; Crabtree 1999), skeletal and cardiac muscle growth and differentiation (Chin et al. 1998; Hughes 1998; Molkentin et al. 1998; Sussman et al. 1998), memory (Mansuy et al. 1998; Winder et al. 1998), and apoptosis (Shibasaki and McKeon 1995; Krebs 1998). Calcineurin is highly conserved in fungi and animals, becoming activated on binding calcium and calmodulin when cytosolic calcium rises, and inhibited on binding the immunosuppressants Cyclosporin A and FK506 in complexes with their respective cellular receptors (Liu et al. 1991a; Klee et al. 1998; Hemenway and Heitman 1999). Feedback regulators of calcineurin have not yet been identified in any cell type.

In the budding yeast Saccharomyces cerevisiae, calcineurin regulates gene expression and ion transport in response to calcium signals (Fig. 1A) but the genes encoding calcineurin (CNA1, CNA2, and CNB1) are not essential for viability (Cyert et al. 1991; Kuno et al. 1991; Liu et al. 1991b; Cyert and Thorner 1992; Ye and Bretscher 1992). Calcineurin promotes growth in high calcium environments by dephosphorylating the transcription factor cytoplasmic Tcn $1 p$ (also called Crzlp and Hal8p), which then accumulates in the nucleus and induces expression of the calcium ATPases Pmclp and Pmrlp (Cunningham and Fink 1994b; Cunningham and Fink 1996; Matheos et al. 1997; Stathopoulos and Cyert

${ }^{1}$ Corresponding author.

E-MAIL kwc@jhunix.hcf.jhu.edu; FAX (410) 516-5213.
1997; Mendizabal et al. 1998; Stathopoulos-Gerontides et al. 1999). Calcineurin also appears to inhibit a vacuolar $\mathrm{H}^{+} / \mathrm{Ca}^{2+}$ exchanger Vcxlp by a posttranslational mechanism thereby preventing its function in calcium tolerance (Cunningham and Fink 1996; Pozos et al. 1996). The exogenous inhibitors of calcineurin, FK506 and Cyclosporin A, restore calcium tolerance to pmc1 mutants by permitting Vcxlp function (Cunningham and Fink 1996). We took advantage of this phenomenon to screen for factors that can inhibit calcineurin function when they are overexpressed. We report the identification of a previously uncharacterized family of proteins, conserved in fungi and animals, which can diminish calcineurin function in vivo and can bind and inhibit calcineurin in vitro directly. The Rcn $1 \mathrm{p}$ family may serve as feedback regulators of calcineurin signaling and thereby modulate a variety of calcineurin-dependent functions.

\section{Results}

A genetic screen for endogenous inhibitors of calcineurin

If endogenous inhibitors of calcineurin are produced in yeast, we reasoned that such molecules would confer calcium tolerance phenotypes similar to those observed with FK506 and Cyclosporin A /Cunningham and Fink 1994b). To identify endogenous calcineurin inhibitors or factors that produce them, we screened a high-dosage library of yeast genomic DNA for genes that conferred calcium tolerance to pmc1 mutants in a Vcxlp-dependent manner. From this screen /Cunningham and Fink 1996) we recovered 20 plasmids with overlapping inserts spanning the previously uncharacterized open reading 


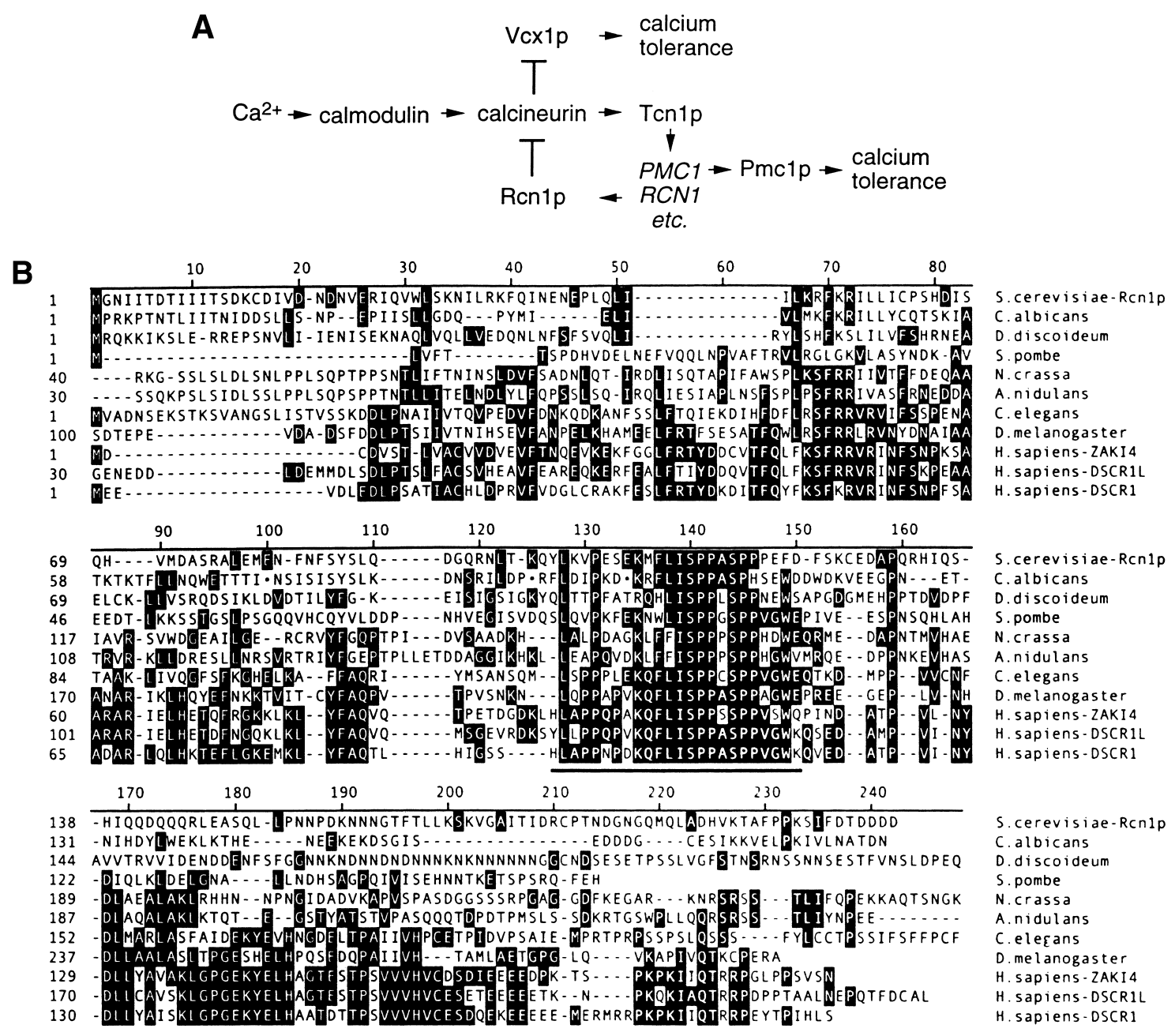

Figure 1. (A) Model of the calcium signaling pathway in yeast. Calcineurin activation by binding calcium and calmodulin inhibits the $\mathrm{H}^{+} / \mathrm{Ca}^{2+}$ exchanger Vcxlp and induces expression of the $\mathrm{Ca}^{2+}$ pump Pmclp through activation of the transcription factor Tcnlp. (B) Multiple sequence alignment of Rcnlp from S. cerevisiae and predicted proteins from other fungi (Candida albicans, Schizosaccharomyces pombe, Neurospora crassa, and Aspergillus nidulans), protozoans (Dictyostelium discoideum), and animals (Caenorhabditus elegans, Drosophila melanogaster, and Homo sapiens) was generated using the Clustal algorithm. Residues identical in at least three of the seven sequences are highlighted and the most conserved central motif corresponding to the DS-24 peptide is underlined. GenBank accession numbers are NP_012763 (S.c.), Q09791 (S.p.), P53806 (C.e.), AAD33987 (D.m.), D83407 (H.s. ZAKI-4), AAF01684 (H.s. DSCR1L), and U85266 (H.s. DSCR1). Other sequences were obtained from ongoing genome sequencing projects (C.a., D.d., and N.c.) or from a compilation of EST sequences at GenBank (A.n. and D.d.). Note that some sequences were truncated at amino- and/or carboxyl termini and three small deletions $(\mathbf{O})$ were introduced into the C. albicans sequence.

frame (ORF) RCN1 (formerly YKL159c, GenBank accession no. Z28159). Subclones containing only the RCN1 gene conferred strong calcium tolerance to a pmc1 mutant but did not confer calcium tolerance to a pmc1 vcx1 double mutant (Fig. 2A) at any concentration (data not shown). The requirement for VCX1 suggested that overexpression of RCN1 promoted calcium tolerance not through buffering or efflux but through the direct or indirect activation of the $\mathrm{H}^{+} / \mathrm{Ca}^{2+}$ exchanger Vcxlp.

If the activation of Vcxlp by RCN1 overexpression was a consequence of calcineurin inhibition, $R C N 1$ overexpression would also be expected to inhibit the activation of Tcn1p, a calcineurin-dependent transcription factor. Indeed, RCN1 overexpression partially blocked calcineurin- and Tcn1p-dependent induction of a PMC1-
lacZ reporter gene (Fig. 2B). A pmc1 vcx1 double mutant was used in this experiment to eliminate any differences in calcium tolerance or sequestration secondary to calcineurin inhibition, though similar effects were observed in wild-type strains (data not shown). No effect of RCN1 overexpression was observed when a constitutively active variant of Tcnlp was coexpressed (Fig. 2B), ruling out the possibility that RCN1 might affect the PMC1 promoter independent of calcineurin. Overexpression of $R C N 1$ also inhibited the expression of a CDRE-lacZ reporter gene (Fig. 2C) containing a single Tcnlp binding site upstream of an inert minimal promoter (Stathopoulos and Cyert 1997). These findings suggest RCN1 overexpression disrupts Tcnlp activation. The ability of $R C N 1$ overexpression to inhibit two independent activi- 
A

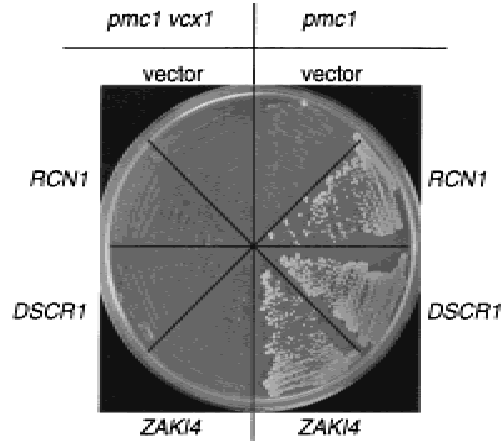

B

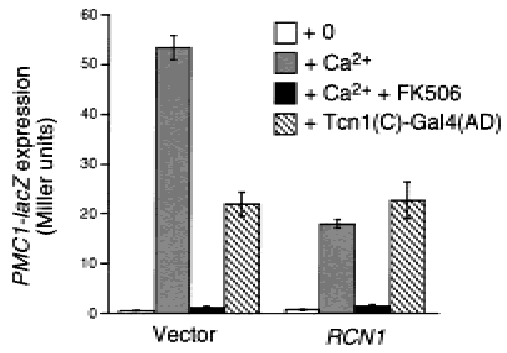

C

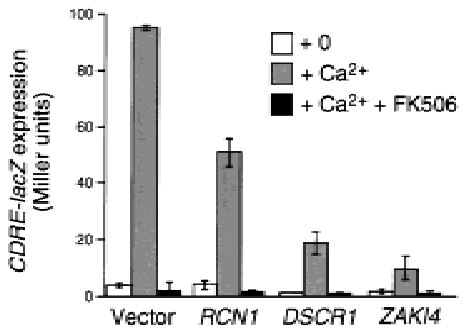

Figure 2. Rcnlp family members inhibit calcineurin function in yeast. (A) Overexpression of yeast RCN1, human DSCR1, or human ZAKI4 genes in yeast blocked calcineurin-dependent inhibition of Vcxlp, thereby restoring growth to pmc1 mutants but not pmc1 vcx1 mutants in high calcium medium (YPD at pH $5.5+200 \mathrm{~mm} \mathrm{CaCl}_{2}$ ). Photographs of colonies were taken after two days incubation at $30^{\circ} \mathrm{C}$. (B) Overexpression of $R C N 1$ inhibited calcineurin-dependent induction of PMC1-lacZ. Reporter gene expression was monitored in pmc1 vcx 1 double mutants carrying either empty vector or RCN1 overexpression plasmid after $4 \mathrm{hr}$ growth in medium supplemented with 100 $\mathrm{mM} \mathrm{CaCl}_{2}$ and $0.3 \mu \mathrm{M}$ FK506 as indicated. Expression was also monitored in wild-type strains containing a constitutively active Tcn $1 \mathrm{p}(\mathrm{C})-\mathrm{Gal} 4(\mathrm{DB})$ transcription factor. $(C)$ Overexpression of RCN1, DSCR1, or ZAKI-4 inhibited expression of $C D R E-1 a c Z$. Betagalactosidase assays were performed as indicated in $B$.

ties of calcineurin suggests that RCN1 exerts its negative effect at the level of calcineurin or further upstream in the calcium-signaling cascade. Experiments described below confirm that the protein product of $R C N 1$ directly binds and inhibits calcineurin in vitro, and therefore is a direct regulator of calcineurin (RCN).

\section{Rcn1p-related proteins}

The RCN1 gene encodes a hydrophilic protein ( $\mathrm{Rcn} 1 \mathrm{p}$ ) of 212 amino acids exhibiting significant homology to pre- dicted proteins in other fungi, invertebrate animals, and mammals (aligned in Fig. 1B) all of which express calcineurin homologs. All proteins in the Rcn $1 \mathrm{p}$ family share a highly conserved central segment containing a novel consensus sequence motif LxxPxxxKxFLISPPxSPPxxW. The human DSCR1 cDNA was identified during analysis of chromosome 21 as a gene mapping near the Down Syndrome critical region (Fuentes et al. 1995). The human ZAKI-4 cDNA was identified in a screen for genes responsive to thyroid hormone in fibroblasts (Miyazaki et al. 1996) and a third closely related human gene termed DSCR1L was recently deposited in GenBank. None of the Rcnlp family members have been characterized functionally.

To determine whether Rcn1p-related proteins retain a similar function, human DSCR1 and ZAKI-4 cDNAs were cloned into yeast expression plasmids and introduced into various yeast strains. As observed with RCN1, overexpression of DSCR1 or ZAKI-4 increased calcium tolerance in pmc1 mutants but not pmc1 vcx1 double mutants (Fig. 2A). Additionally, expression of DSCR1 and ZAKI-4 diminished calcineurin-dependent induction of $P M C 1-1 a c Z$ by $84 \%$ and $95 \%$, respectively, and also strongly inhibited induction of the CDRE-lacZ reporter gene (Fig. 2C). Thus, despite their limited sequence similarity to Rcn1p, DSCR1 and ZAKI-4 retained the ability to inhibit calcineurin function when expressed in yeast.

\section{Rcn1p and DSCR1 bind and inhibit calcineurin in vitro}

Rcnlp and DSCR1 were expressed as glutathionine $S$ transferase (GST) fusion proteins in Escherichia coli, purified by affinity chromatography, and tested for interactions with calcineurin purified from bovine brain. Bovine calcineurin specifically bound to both GST-DSCR1 and GST-Rcn1p on glutathione-agarose beads (Fig. 3A), though the crosskingdom interaction between Rcn $1 \mathrm{p}$ and calcineurin appeared weaker. Calcineurin bound to GST-DSCR 1 in buffers containing either 2 mM EGTA or $2 \mathrm{mM} \mathrm{CaCl}_{2}+$ calmodulin (Fig. 3B) and therefore was independent of the calcium concentration. Furthermore, FK506/FKBP12 complexes exhibited a calmodulin-dependent interaction with the DSCR1/calcineurin complex (Fig. 3C). These results suggest DSCR1 binds calcineurin at a site (or sites) distinct from those that bind calmodulin and FK506/FKBP12.

The functional significance of DSCR1 and Rcn1p interaction with calcineurin was investigated using standard protein phosphatase assays with phospho-RII peptide as substrate. Addition of purified GST-DSCR1 strongly inhibited the calcium/calmodulin-dependent dephosphorylation of RII peptide by bovine calcineurin, whereas the same quantities of GST had no effect on calcineurin activity (Fig. 3D). Increasing calmodulin concentration 10 -fold did not overcome inhibition by GSTDSCR1, confirming that DSCR1 does not compete with calmodulin. Much higher levels of GST-Rcn1p were required to inhibit bovine calcineurin (Fig. 3E) consistent with its lower binding (Fig. 3A). Finally, a synthetic 24- 
Figure 3. Binding and inhibition of calcineurin in vitro by Rcn 1 p, DSCR 1 , and DS24 peptide. (A) GST, GST-Renlp, and GST-DSCR1 were immobilized on glutathione-sepharose beads and assayed for the ability to bind purified bovine brain calcineurin. (B) Immobilized GST-DSCR1, but not GST alone bound calcineurin in buffer A, or buffer A supplemented with either $2 \mathrm{mM} \mathrm{CaCl}_{2}$ or $2 \mathrm{~mm}$ EGTA. (C) GSTDSCR1/ calcineurin bound to FK506/ FKBP12 in the presence of calmodulin and $2 \mathrm{mM} \mathrm{CaCl}_{2}$. $(D-F)$ Dephosphorylation of RII peptide by calcineurin was inhibited by purified GST-DSCR1, GST-Ren1p (100 $\mu \mathrm{g} / \mathrm{ml})$, and DS-24 synthetic peptide. Tenfold excess calmodulin failed to reverse calcineurin inhibition by DSCR1.
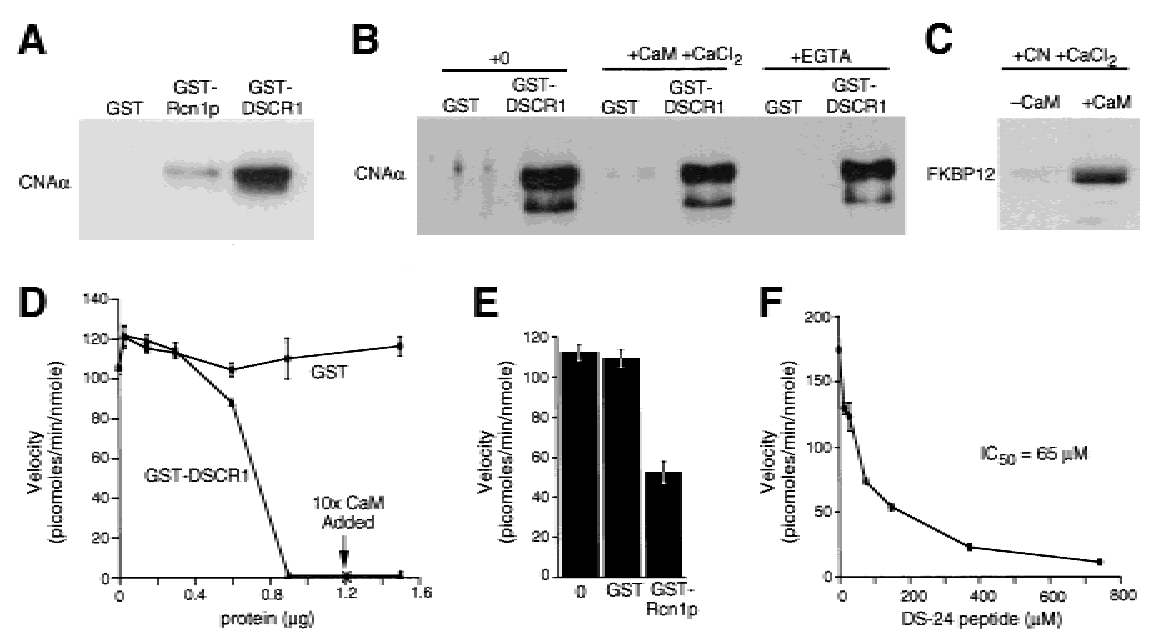

residue peptide surrounding the most conserved motif of DSCR1 (peptide DS-24) also inhibited calcineurin in a dose-dependent manner (Fig. 3F). The DS-24 peptide was much less potent than full-length DSCR1, suggesting that other regions of DSCR1 also contribute to interactions with calcineurin. These results demonstrate that two divergent members of the Rcn $1 \mathrm{p}$ family of proteins can directly bind and inhibit calcineurin.

\section{Calcineurin-dependent expression of Rcn1p}

Calcium signals increased the expression of DSCR1 mRNA in mammalian cell lines (Crawford et al. 1997; Leahy et al. 1999; Fuentes et al. 2000). Therefore, we tested whether RCN1 transcription and Rcn $1 \mathrm{p}$ accumulation was regulated in yeast. A RCN1-lacZ reporter gene containing $2-\mathrm{kb}$ of the $R C N 1$ promoter region was expressed at low levels in wild-type cells during growth in standard medium and was induced $>20$-fold after shift to high calcium conditions (Fig. 4A). Induction of RCN1lac $Z$ in response to calcium was completely blocked by addition of FK506 or by deletion of the genes encoding Tcn 1p (Fig. 4A) or calcineurin (data not shown), suggesting RCN1 may be another downstream target of the calcineurin-dependent transcription factor. Western blot analysis of cells expressing an epitope-tagged Rcn1p-HA protein from a low-dosage plasmid confirmed this pattern of expression. Growth in high calcium stimulated Rcn1p-HA accumulation in wild-type cells (Figs. 4B, lanes 1,2 ) but not in tcn 1 mutants (lanes 5,6). Induction of Rcn1p-HA was also blocked in cna1 cna2 double mutants that lack the two catalytic A subunits of calcineurin (not shown), in cnb1 mutants that lack the single regulatory B subunit of calcineurin (Fig. 4C, lanes 9,10), and in cmd1-6 mutants that express a defective calmodulin (Fig. 4C, lanes 5,6) that is unable to bind calcium or activate calcineurin (Geiser et al. 1991). Thus, calcium signaling through calmodulin, calcineurin, and Tcn $1 \mathrm{p}$ was required for stimulation of Rcnlp expression above a basal level. Strong up-regulation of Rcnlp in yeast and DSCR1 in mammals in response to calcineurin signaling may therefore constitute a negative-feedback mechanism modulating calcineurin activity in vivo.

An additional effect of calcineurin on Rcn $1 p$ stability was revealed through analysis of Rcnlp-HA levels in calcineurin-deficient mutants. In nonsignaling conditions, accumulation of Rcnlp-HA was greatly reduced in cnb1 mutants and cna1 cna2 double mutants, relative to wild-type and tcn 1 mutants and cmd1-6 mutants (Fig. $4 B, C$; data not shown). These results reveal a role of calcineurin A/B heterodimers on Rcnlp stability or basal expression that is independent of calcium, calmodulin, and Tcn 1 p. Basal expression of RCN1-lacZ was identical in wild-type and cnb1 mutants, suggesting that Rcn1p stability might be increased by the presence of calcineurin in the cell (data not shown). Stability of Rcnlp-HA was difficult to quantitate in cnb1 mutants because of the low initial levels of the protein. However, we noticed that addition of FK506 plus calcium caused Rcn1p-HA levels to decline in wild type, tcn 1 mutants, and cmd1-6 mutants to levels approaching that of $c n b 1$ mutants whereas FK506 alone had no effect (Fig. 4B,C). Treatment with FK506 plus calcium accelerated the disappearance of Rcnlp-HA in wild-type cells that had been pretreated with cycloheximide to inhibit protein synthesis (Fig. 4D). These findings demonstrate that calcineurin stabilizes Rcn $1 p$ in vivo, even in the absence of activation by $\mathrm{Ca}^{2+} /$ calmodulin.

\section{The phenotype of ren1 null mutants}

The above results all suggest that Rcn $1 \mathrm{p}$ may operate as a feedback inhibitor of calcineurin signaling in vivo. If this were the only role of Rcnlp in yeast, mutants lacking Rcn $1 p$ would likely exhibit characteristics of enhanced calcineurin activity. To test this hypothesis, a rcn1 null mutant was constructed by homologous recombination and assayed for Tcnlp activation and Vcxlp inhibition. Surprisingly, both assays revealed reduced calcineurin activity in rcn1 null mutants. First, the low calcium tolerance of pmc1 mutants but not pmc1 vcx1 double mutants was suppressed partially by 
A

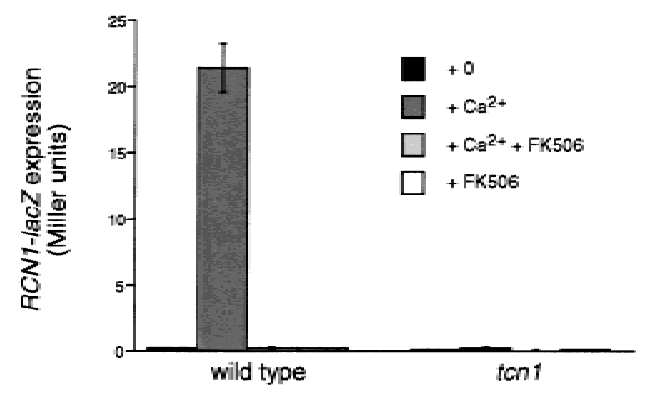

B

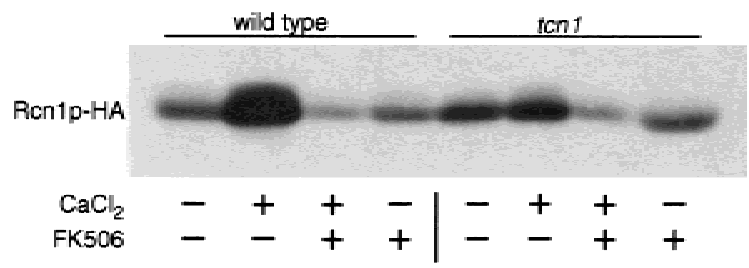

C

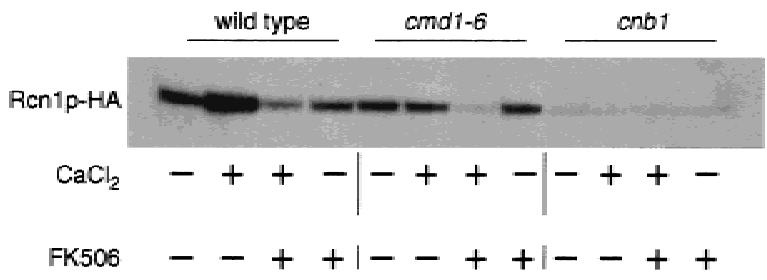

D

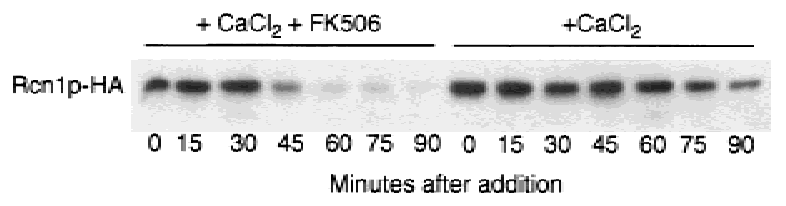

Figure 4. Calcineurin regulates $R C N 1$ transcription and Rcn $1 \mathrm{p}$ stability in yeast. $(A)$ Induction of a RCN1-lacZ reporter gene required calcium, calcineurin, and Tcnlp. A RCN1-lacZ reporter gene was introduced into wild-type and $t c n 1$ mutants. $\beta$-Galactosidase activity was assayed in three independent transformants following $4 \mathrm{hr}$ growth at $30^{\circ} \mathrm{C}$ in YPD at $\mathrm{pH} 5.5$ medium supplemented with $100 \mathrm{mM} \mathrm{CaCl}_{2}$ and $0.3 \mu \mathrm{M}$ FK506 as indicated. (B) Rcn 1p-HA protein levels increased in response to calcium via the activation of calcineurin and Tcnlp. A lowdosage plasmid containing the epitope-tagged $R c n 1 p-H A$ gene was introduced into wild-type yeast and tcn 1 mutants. Cells were grown as in $A$ and then total cell protein was fractionated by SDS-PAGE and analyzed by Western blotting. $(C)$ Basal accumulation of Rcnlp-HA required calcineurin but not calcium, calmodulin, or Tcnlp. Total cell extracts were prepared and analyzed as in $B$ using wild-type cells, cnb1 mutants lacking the regulatory B subunit of calcineurin, or cmd1-6 mutants lacking $\mathrm{Ca}^{2+}$-binding sites in calmodulin. (D) FK506 destabilized Rcn1p-HA in high calcium conditions. Wild-type yeast expressing Rcn1p-HA were treated with $100 \mu \mathrm{M}$ cycloheximide for 20 min and then treated with $100 \mathrm{mM} \mathrm{CaCl}_{2}$ in either the presence or absence of FK506. Total cell protein was extracted at $15 \mathrm{~min}$ intervals and analyzed by Western blotting as in $B$.

the deletion of RCN1 (Fig. 5A). The addition of FK506 increased the calcium tolerance of pmc1 mutants to the same level with or without Rcn $1 p$, indicating that Rcnlp and calcineurin function within a common pathway. Secondly, calcineurin-dependent induction of PMC1-
lacZ, RCN1-lacZ, and FKS2-lacZ in rcn1 mutants decreased by $93 \%, 87 \%$, and $62 \%$, respectively, relative to wild type. Similar effects were observed in pmc1 vcx1 double mutants (Fig. 5B). Induction of CDRE-lacZ was also largely dependent on Rcn $1 p$ (Fig. 5C) but expression of a calcineurin-independent CYC1-lacZ reporter was unaffected by Rcnlp function (Fig. 5B). Calcineurin-dependent dephosphorylation of Tcn $1 \mathrm{p}$ causes a shift in its mobility on SDS gels even in wild-type cells (Stathopoulos and Gerontides 1999). Using this method, we found that epitope-tagged Tcnlp-HA from $r c n 1$ mutants migrated similar to that of cnb1 mutants (Fig. 5D), indicating that $\mathrm{Rcn} 1 \mathrm{p}$ was required for calcineurin-dependent dephosphorylation of Tcnlp. Thus, at least two independent outputs of calcineurin were specifically impaired in rcn1 mutants. Interestingly, the positive role of Rcnlp on calcineurin may also be conserved in mammalian cells because expression of DSCR1 in rCn1 mutants partially complemented the defect in PMC1-lacZ expression (Fig. 5B).

The nature of Rcnlp's positive contribution to calcineurin signaling was investigated further by monitoring calcineurin expression and stability. Neither deletion nor overexpression of Rcnlp affected expression of an epitope-tagged Cnalp-MYC protein in nonsignaling conditions (Fig. 6A). In high calcium conditions however, Cnalp-MYC consistently declined to lower levels in rcn1 mutants compared to wild type and remained higher in Rcnlp-overexpressing strains. The calcium-dependent decline of Cnalp-MYC was more pronounced in pmc1 vcx1 mutants (Fig. 6B) where cytosolic calcium increases to higher levels than wild type (Miseta et al. 1999). Inhibition of calcineurin through FK506 addition did not prevent the loss of Cnalp-MYC in the presence of high calcium. To determine if Rcnlp affected CnalpMYC stability, Cnalp expression was assayed in the presence of cycloheximide. Surprisingly, cycloheximide blocked the down-regulation of Cnalp-MYC with or without Rcn1p and/or FK506 (Fig. 6C; data not shown). Thus, Cnalp stability appeared to be insensitive to calcium, FK506, and Rcnlp. Instead, the elevated accumulation of Cnalp-MYC caused by Rcnlp during signaling conditions may reflect a positive effect of Rcnlp on calcineurin expression. This hypothesis was confirmed through analysis of CNA1-lacZ, CNA2-lacZ, and CNB1-lacZ expression (Fig. 7). In standard medium with or without calcium and FK506, these reporter genes were expressed respectively at $\sim 50 \%, \sim 35 \%$, and $\sim 150 \%$ higher levels in wild-type cells relative to $r c n 1$ mutants. Interestingly, high calcium conditions diminished expression of all three reporter genes in a FK506-sensitive fashion. These findings reveal a significant role for Rcn $1 p$ in stimulating calcineurin expression. This effect provides at least a partial explanation for the positive role of Rcnlp on calcineurin signaling in yeast.

\section{Discussion}

This study reports the identification of a conserved family of proteins that appear to function as feedback inhibi- 
Figure 5. Rcnlp promotes calcineurin function and expression. (A) Rcnlp promotes calcineurin-dependent inhibition of Vcxlp. The optical density of pmc1 mutant cultures grown for $20 \mathrm{hr}$ at $30^{\circ} \mathrm{C}$ was measured at $650 \mathrm{~nm}$ and plotted as a function of added $\mathrm{CaCl}_{2}$. Solid lines represent growth of pmc1 or rcn1 pmc1 mutants and dashed lines represent growth of $p m c 1 \mathrm{vcx} 1$ or $r c n 1$ pmc1 vcx1 mutants in the presence and absence of $0.3 \mu \mathrm{M}$ FK506. (B) Calcineurin-dependent induction ratios were calculated for various reporter genes expressed in pmc1 $v c x 1$ double mutants or $r c n 1$ pmc1 vcx 1 triple mutants as indicated. Expression of DSCR1 partially complemented the rcn1 defect for PMC1-lacZ expression. $(C)$ Induction of the calcineurin-dependent minimal CDRE-lacZ reporter gene was reduced in the absence of Rcnlp. The CDRE-lacZ reporter gene was introduced into pmc1 $v c x 1$ and rcn1 pmc1 vcx1. $\beta$-Galactosidase activity was assayed in three independent transformants following $4 \mathrm{hr}$ growth at $30^{\circ} \mathrm{C}$ in YPD medium at pH 5.5 supplemented with $100 \mathrm{~mm} \mathrm{CaCl}_{2}$ and $0.3 \mu \mathrm{M}$ FK506 as indicated. (D) Western blot analysis of Tcn1p-HA extracted from wild type and rcn1 mutants. Total protein was extracted from log phase cells grown in YPD medium at pH 5.5 and analyzed by Western blotting using 12CA5 monoclonal antibody.

tors of calcineurin during calcium signaling. Recombinant Rcn1p and DSCR1 proteins bound and inhibited bovine calcineurin activity in vitro while overexpression of Rcnlp and DSCR1 inhibited at least two independent functions of yeast calcineurin in vivo, including the activation of Tcnlp and the inhibition of Vcxlp (see Fig. 1A). RCN1 transcription and Rcnlp accumulation in yeast were strongly induced by calcineurin-dependent activation of Tcnlp, supporting the hypothesis that Rcnlp operates as an endogenous feedback inhibitor of calcineurin signaling. Recent studies suggest that DSCR1 functions as a feedback inhibitor of calcineurin signaling in human cells. DSCR1 transcription in human astrocytoma cells was strongly stimulated by calcineurin signaling and DSCR1 overexpression inhibited calcineurin-dependent activation of NFAT (Rothermel et al. 2000; Fuentes et al. 2000). Together these findings suggest broad conservation of the Rcnlp-related proteins as feedback inhibitors of calcineurin.

The analysis of $r c n 1$ null mutants also revealed a stimulatory role of Rcnlp on calcineurin signaling. Calcineurin-dependent regulation of both Tcnlp and Vcxlp was clearly reduced in $r c n 1$ mutants but not completely abolished as judged by the more severe consequences of adding FK506. The apparent deficiency of calcineurin signaling in $r c n 1$ mutants may be the result of decreased calcineurin expression. For example, Cnalp levels were lower in $r c n 1$ mutants and higher in Rcn1p-overexpressing strains as compared to wild-type strains grown in high calcium conditions. We detected no obvious effects of Rcnlp on either Cnalp accumulation in nonsignaling conditions or on Cnalp stability in any conditions tested. Additionally, we could not detect any effect of Rcnlp on Vcxlp or Tcnlp function when calcineurin had been inactivated by FK506, although Rcn1p is unstable under these conditions. The simplest model consistent with these results is one where Rcnlp stimulates calcineurin expression during calcium signaling.

Calcineurin expression in yeast has not yet been studied in detail. Rcnlp increased expression of CNA1, $C N A 2$, and CNB1 reporter genes whereas calcium decreased expression through an FK506-sensitive mechanism (Fig. 7). Cnalp levels also declined during growth in high calcium conditions, an effect that was enhanced in rcn1 mutants (Fig. 6) and diminished in cmd1-6 mutants (data not shown). These results suggest calcineurin activation may down-regulate expression of its structural genes, an effect that would be stimulated by calmodulin and inhibited by Rcnlp. However, the decline of Cnalp was not blocked by FK506 addition as if another calcium-dependent mechanism contributed to calcineurin down-regulation in yeast. Therefore, calcineurin expression, accumulation, and function appear to be regulated by calcium at multiple levels. Further analysis of calcineurin dynamics is warranted in order to understand the significance of this unexpected complexity and to fully explain the calcineurin-deficient phenotype of $r \mathrm{cn} 1 \mathrm{mu}-$ tants.

Additional roles for Rcnlp in promoting calcineurin function in yeast can not be ruled out. For example, 
A

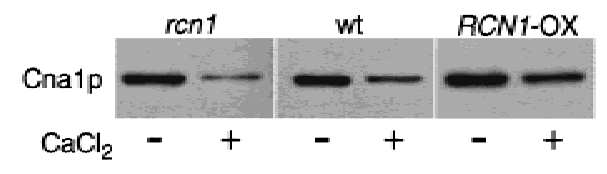

B
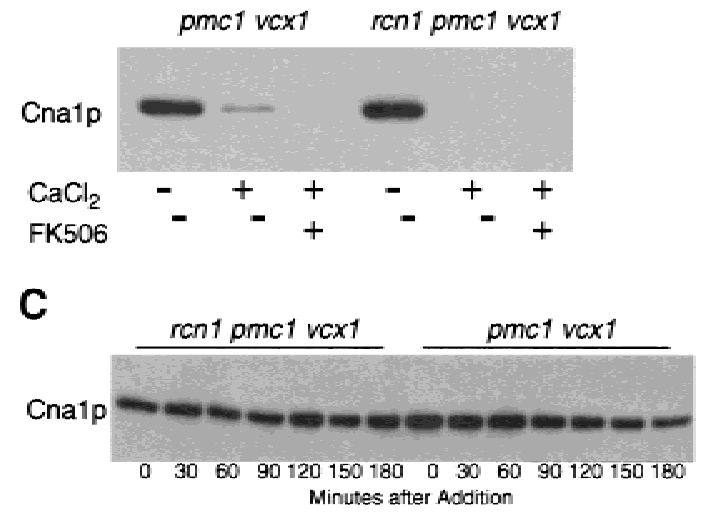

Figure 6. Down-regulation of calcineurin during calcium signaling conditions. (A) Cnalp expression correlates with Rcnlp in high calcium conditions. Western blots of Cnalp-MYC in rcn1 mutants, wild type, and Rcnlp-overexpressing strains were performed on total cell protein after $4 \mathrm{hr}$ growth in YPD medium at pH 5.5 supplemented with $100 \mathrm{~mm} \mathrm{CaCl}_{2}$ as indicated. (B) Endogenous Rcnlp increases Cnalp expression in pmc1 $v c x 1$ mutants in high calcium conditions. Experimental conditions were as described in $A$ except $0.3 \mu \mathrm{M}$ FK506 was added as indicated. $(C) \mathrm{Rcn} 1 \mathrm{p}$ is not required to stabilize Cnalp. CnalpMYC levels were monitored in pmc1 vcx1 double and rcn1 pmc1 vcx1 triple mutants after a pretreatment with $100 \mu \mathrm{M}$ cycloheximide for $20 \mathrm{~min}$ followed by addition of $100 \mathrm{mM} \mathrm{CaCl}_{2}$ and $0.3 \mu \mathrm{M}$ FK506. Total cell protein was extracted at $30 \mathrm{~min}$ intervals and analyzed by Western blotting as in Figure 3.

Rcn1p may actually stimulate calcineurin signaling to some degree in vivo through a mechanism that was not reconstituted or detectable in our in vitro assays, even at levels 100-fold lower than those required to inhibit calcineurin activity. Low doses of these proteins might increase calcineurin activity in vivo but such effects might escape detection in vitro if oxidative inactivation of calcineurin was also stimulated (Wang et al. 1996). Alternatively, these proteins might promote interactions between calcineurin and its natural substrates, much like the targeting or scaffolding subunits of type 1 protein phosphatase (Hubbard and Cohen 1993; Sim and Scott 1999). For example, the inhibitor-2 proteins prevent some activities of PP1 while stimulating others (Alessi et al. 1993), possibly by altering the partitioning of active PP1 molecules. Remarkably, genetic analysis of inhibitor-2 function in yeast revealed positive and negative effects on PP1 function (Tung et al. 1995) much like the effects of Rcnlp on calcineurin function reported here. Two proteins in mammals, AKAP79 (Coghlan et al. 1995; Kashishian et al. 1998) and Cabin1/cain (Lai et al. 1998; Sun et al. 1998; Youn et al. 1999), are known to bind calcineurin at sites distinct from the FK506/
FKBP12 binding sites, to inhibit calcineurin phosphatase activity, and to also bind other cellular factors which may include substrates. The calcineurin-binding domains of both proteins are rather basic in character and not obviously related to the conserved domains of the Rcnlp family members. These structural differences and the dramatic up-regulation of Rcn1p and DSCR1 in response to calcineurin signaling distinguish this new family of calcineurin regulators from proteins described previously.

Finally, the possibility that Rcn1p and DSCR1 act as downstream effectors of calcineurin signaling remains to be fully explored. We have been unable to detect any effect of Rcn 1 p on Vcxlp or Tcn $1 p$ function in various contexts, but negative results of this nature do not rule out the possibility that $\mathrm{Rcn} 1 \mathrm{p}$ mediates the regulation of other factors that respond to calcineurin signaling. The most conserved segment KxFLISPPXSPPx bears some resemblance to the conserved SPxxSPxxSPxx motifs repeated several times in NFAT proteins (Rao et al. 1997).

A

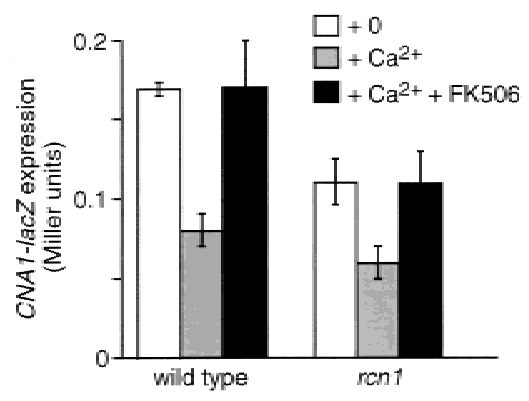

B
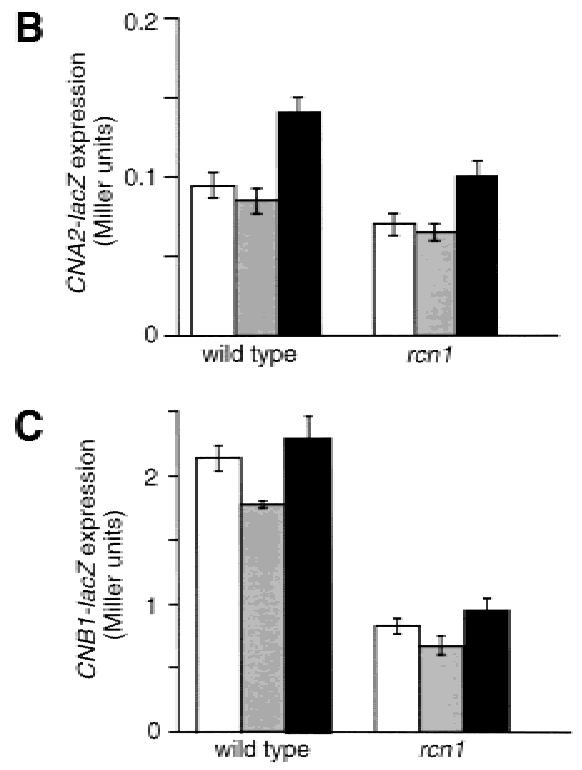

Figure 7. Involvement of $\mathrm{Rcn} 1 \mathrm{p}$ in expression of calcineurin structural genes. (A) CNA1-lacZ, (B) CNA2-lacZ, and $(C)$ $C N B 1-l a c Z$ reporter genes were introduced into wild type and ren 1 deletion mutant. $\beta$-Galactosidase activity was assayed in three independent transformants following $4 \mathrm{hr}$ growth at $30^{\circ} \mathrm{C}$ in YPD medium at pH 5.5 supplemented with $100 \mathrm{mM} \mathrm{CaCl}_{2}$ and $0.3 \mu \mathrm{M}$ FK506 as indicated. 
Table 1. S. cerevisiae strains used in this study

\begin{tabular}{|c|c|c|}
\hline Strains & Genotype & Reference \\
\hline DMY14 & $\operatorname{tcn} 1:: G 418$ & $\begin{array}{l}\text { Matheos et al. } \\
\text { (1997) }\end{array}$ \\
\hline JGY148 & cmd1-6 & $\begin{array}{l}\text { Moser et al. } \\
(1996)\end{array}$ \\
\hline K482 & pmc1::TRP1 & $\begin{array}{l}\text { Cunningham and } \\
\text { Fink (1994) }\end{array}$ \\
\hline K537 & cna1::URA3 cna2::HIS3 & this study \\
\hline K601 & + & $\begin{array}{l}\text { Cunningham and } \\
\text { Fink (1994) }\end{array}$ \\
\hline K603 & cnb1::LEU2 & $\begin{array}{l}\text { Cunningham and } \\
\text { Fink (1994) }\end{array}$ \\
\hline K651 & pmc1::TRP1 vcx1s & $\begin{array}{l}\text { Cunningham and } \\
\text { Fink (1996) }\end{array}$ \\
\hline K665 & pmc1::TRP1 vcx1s & $\begin{array}{l}\text { Cunningham and } \\
\text { Fink (1996) }\end{array}$ \\
\hline TKY268 & rcn1::HIS3 pmc1::TRP1 & this study \\
\hline TKY275 & rcn1::HIS3 & this study \\
\hline TKY278 & rcn1::HIS3 pmc1::TRP1 vcx1s & this study \\
\hline
\end{tabular}

All strains are isogenic to W303-1A (ade2-1 can1-100 his3-11, 15 leu2-3, 112 trp1-1 ura3-1).

These SP repeats are thought to be functionally important for NFAT regulation by calcineurin, serving as substrates of calcineurin after phosphorylation by protein kinases in the nucleus (Beals et al. 1997; Chow et al. 1997; Zhu et al. 1998; Crabtree 1999). Therefore, it is conceivable that calcineurin activation dephosphorylates Rcn1p family members as a mechanism for regulating additional downstream factors. Because Rcnlp stability and expression depend on interactions with calcineurin, any effector functions of Rcnlp would also be affected by FK506.

The findings reported here and elsewhere (Fuentes et al. 2000) suggest that feedback inhibition of calcineurin is conserved from yeast to humans. The function of this feedback mechanism may be to fine-tune calcineurin signaling over a spectrum of intervals and conditions. Improper regulation of Rcnlp family members might lead to the disruption of calcineurin function in humans and contribute disease. For example, the increased dosage of DSCR1 in trisomy-21 individuals may contribute to the neurological, cardiac, or immunological defects observed in Down syndrome patients (Epstein 1995) through inhibition of calcineurin signaling. It will be interesting to determine if the interactions between DSCR1 and calcineurin in human cells are as complex as those we have observed for Rcn $1 \mathrm{p}$ in yeast. It is not yet known if endogenous levels of DSCR1 are required to promote calcineurin function in vivo, or if calcineurin expression is down-regulated during prolonged calcium signaling. If FK506 and Cyclosporin A destabilize DSCR1 or its homologs in human cells, the efficacy or side effects of these drugs in transplantation therapies might be attributed to loss of these proteins. Understanding the relationship between the human Rcnlp family members and calcineurin in vivo will not only enhance our understanding of calcineurin function but also potentially provide novel therapeutic targets to control calcineurin function in humans.

\section{Materials and methods \\ Genetic procedures}

A library of yeast genomic DNA carried on high-dosage plasmids was screened for potential inhibitors of calcineurin by selecting for plasmids that could restore growth of a pmc1 null mutant (strain $\mathrm{K} 473$ ) on solid YPD medium at $\mathrm{pH} 5.5$ supplemented with $200 \mathrm{~mm} \mathrm{CaCl}_{2}$ (Cunningham and Fink 1996). Of 24 plasmids that were recovered, two carried the $\mathrm{Ca}^{2+}$ pumps Pmclp and Pmrlp, two carried Vcxlp, and 20 carried the uncharacterized yeast gene $R C N 1 / Y K L 159 c$ plus flanking sequences. Subcloning demonstrated the active gene was $R C N 1$. The entire RCN1 coding sequence was deleted from the genome by homologous recombination using plasmid pTJK39 linearized by EcoRI digestion. The resulting rcn1::HIS3 null mutant was crossed to other mutants in an isogenic background (Matheos et al. 1997) to generate strains bearing multiple mutations (see Table 1).

\section{Recombinant DNA}

All recombinant DNA work was conducted with standard techniques with enzymes purchased from New England Biolabs or GIBCO BRL. The ren1::HIS3 disruption plasmid pTJK39 was constructed by sequentially ligating two PCR products corresponding to the $5^{\prime}$ and $3^{\prime}$ flanking regions of $R C N 1$ that had been amplified from genomic DNA using the primers CCGAATTCGCCATACTATCAAATG and GGGGATCCCTGCAGTTCTGTGTTT (for 5' sequences) and CCCTCGAGGATGGCGAGGCGATTTG and CCGAATTCGAATAGTAATAAAGAT (for $3^{\prime}$ sequences) into vector pRS303 digested with $E c o R I+B a m H I$ and EcoRI + XhoI, respectively. The RCN1-HA expression plasmid pTJK29 was generated by subcloning the 3xHA tag from pBSHA3 (Cunningham and Fink 1994a) into pRS316 (Sikorski and Hieter 1989) containing 5' regulatory and coding sequences from the $R C N 1$ gene lacking a stop codon that had been amplified by PCR using primers GATCTTCACAAATCTTGGGG and GCCATCTTATCTAGAATCATCGTCATCAG. A downstream stop codon was reconstructed by generating a frameshift at the SpeI site in the polylinker. Plasmids bearing CNA1-lacZ, CNA2-lacZ, CNB1-lacZ, and RCN1-lacZ reporter genes were constructed by subcloning PCR-generated DNA segments corresponding to nucleotides -2000 and +3 relative to the initiator codon of each gene into plasmid pLG $\Delta 178$ using the following primers: for CNA1, CTCGAGAACGGAAGTGGCAACTTG and GGATCCCATTGGCGTTGAGAGTGT; for CNA2, CTCGAGTAAAGCTGGAGCCAAGAC and GGATCCCATTGCGGGTTCAAGAAG; for CNB1, CTCGAGGACTAGTTCAAAGGTAAA and AGATCTCATTTTAAGAAATAAAAATGC; and for RCN1, CTGCTCGAGCAGAAAATTCGTGAAC and GGGATCCCATCTGCAGTTCTGTGT. The ZAKI4 expression plasmid pTJK1 was constructed by subcloning the XmnI-NsiI fragment of phZAKI-4-3.2 (courtesy of H. Seo, Nagoya University, Japan) into pRS425MET digested with SmaI + PstI. The DSCR1 expression plasmid pTJK37 was constructed by subcloning a PCR product from DSCR1-1pBS (courtesy of X. Estivill, I.R.O., Barcelona, Spain) using primers GCGAGGATCCGTATGGAGGAGGTGGACCTG and CCCTCACTCGAGGCTGAGGTGGATCGGCGTGTA into pRS426MET digested with BamHI + XhoI. The GST-DSCR1 expression plasmid pTJK92 was constructed from the same PCR product after subcloning a BamHI-BgIII fragment into the BamHI site of pGEX3X. The GST-Rcnlp expression plasmid 
pTJK93 was constructed by subcloning into the BamHI site of pGEX-3X a BgIII + BamHI-digested PCR product using primers CTGCAGGGGATCCGTATGGGTAATATTATAAC and CGAAATAGATCTGATGAAGAGGAGGT. The Cnalp-MYC expression plasmid pTJK91 was constructed by subcloning the $3 \times$ MYC sequences from pKB241 into pRS315 containing the promoter and coding sequences of CNA1 that had been amplified using the primers CTCGAGAACGGAAGTGGCAACTTG plus GGATCCCATTGGCGTTGAGAGTGT and AGATCTAGAATGTCGAAAGACTTGAATTCT plus GGCTGATGGTGGTGTTCA. Plasmid pAMS342 (Stathopoulos and Cyert 1997) harboring the CDRE-lacZ reporter gene and pDM16 harboring the activated TCN1 allele (Matheos et al. 1997) were described previously.

\section{Protein analysis and purification}

The consensus Aspergillis nidulans and Dictyostelium discoideum sequences were compiled from overlapping cDNA and gDNA sequences at GenBank and the $D$. discoideum Genome Project. Protein sequences were aligned using the Clustal program from DNAstar based on the PAM250 weight table. GSTDSCR1, GST-Rcn1p, and GST were expressed in TOPP2 cells as suggested by the manufacturer (Stratagene) and purified on glutathione-agarose beads according to instructions (Pharmacia Biotech). $\beta$-Galactosidase assays were conducted as described previously (Cunningham and Fink 1996). The average $\beta$-galactosidase activity measured from three independent transformants is plotted ( \pm S.D. $)$.

Log-phase cell cultures were harvested, extracted with trichloroacetic acid, and processed for SDS-PAGE, Western blotting, and ECL detection as described previously (Cunningham and Fink 1996). Monoclonal antibodies 12CA5 (Boehringer) or 9E10 (Santa Cruz Biotechnology) were used to detect HA and MYC-tagged proteins respectively. Calcineurin was detected using polyclonal antibodies (gift of Claude Klee, NIH). For studies of Rcnlp-HA stability, yeast cells were grown to log phase overnight in SC minus uracil medium and then shifted to YPD medium at $\mathrm{pH} 5.5$. After $1 \mathrm{hr}$ incubation, $100 \mu \mathrm{g} / \mathrm{ml}$ cycloheximide was added and cultures were split and diluted into the same medium containing $100 \mathrm{~mm} \mathrm{CaCl}_{2}$ with or without $0.3 \mu \mathrm{M}$ FK506. Samples were processed for Western blotting as described above.

Purified bovine brain calcineurin, calmodulin, and FKBP12 were obtained from Sigma Chemical Corp. Polyclonal antiFKBP12 was from BIOMOL. Calcineurin binding experiments were performed as described (Lai et al. 1998) followed by SDSPAGE and Western blot detection. Calcineurin activity assays using ${ }^{32} \mathrm{P}$-labeled RII peptide as substrate were performed as described (Fruman et al. 1996; Sagoo et al. 1996) except $25 \mathrm{~nm}$ calcineurin was used in $15 \mathrm{~min}$ reactions at $30^{\circ} \mathrm{C}$. The DS-24 peptide HLAPPNPDKQFLISPPASPPVGWKC was synthesized at Pocono Rabbit Farm and Laboratory.

\section{Acknowledgments}

We are grateful to Claude Klee for antibodies, to Xavier Estivill, Martha Cyert and Hisao Seo for plasmids, to Fujisawa USA Inc. for gifts of FK506, and to Liz O'Sullivan for technical assistance. This research was jointly supported by the Basil O'Connor Starter Scholar Research Award (FY96-1131) from the March of Dimes Birth Defects Foundation, the Searle Scholars Program/ The Chicago Community Trust, a Research Grant from the National Institutes of Health (GM53082) and a grant from the American Heart Association.

The publication costs of this article were defrayed in part by payment of page charges. This article must therefore be hereby marked "advertisement" in accordance with 18 USC section 1734 solely to indicate this fact.

\section{References}

Alessi, D.R., Street, A.J., Cohen, P., and Cohen, P.T. 1993. Inhibitor-2 functions like a chaperone to fold three expressed isoforms of mammalian protein phosphatase-1 into a conformation with the specificity and regulatory properties of the native enzyme. Eur. J. Biochem. 213: 1055-1066.

Beals, C.R., Sheridan, C.M., Turck, C.W., Gardner, P., and Crabtree, G.R. 1997. Nuclear export of NF-ATc enhanced by glycogen synthase kinase-3. Science 275: 1930-1934.

Chin, E.R., Olson, E.N., Richardson, J.A., Yang, Q., Humphries, C., Shelton, J.M., Wu, H., Zhu, W., Bassel-Duby, R., and Williams, R.S. 1998. A calcineurin-dependent transcriptional pathway controls skeletal muscle fiber type. Genes \& Dev. 12: 2499-2509.

Chow, C.W., Rincon, M., Cavanagh, J., Dickens, M., and Davis, R.J. 1997. Nuclear accumulation of NFAT4 opposed by the JNK signal transduction pathway. Science 278: 1638-1641.

Coghlan, V.M., Perrino, B.A., Howard, M., Langeberg, L.K., Hicks, J.B., Gallatin, W.M., and Scott, J.D. 1995. Association of protein kinase $\mathrm{A}$ and protein phosphatase $2 \mathrm{~B}$ with a common anchoring protein. Science 267: 108-111.

Crabtree, G.R. 1999. Generic signals and specific outcomes: Signaling through $\mathrm{Ca}^{2+}$, calcineurin, and NF-AT. Cell 96: 611-614.

Crawford, D.R., Leahy, K.P., Abramova, N., Lan, L., Wang, Y., and Davies, K.J. 1997. Hamster adapt78 mRNA is a Down syndrome critical region homologue that is inducible by oxidative stress. Arch. Biochem. Biophys. 342: 6-12.

Cunningham, K.W. and Fink, G.R. 1994a. $\mathrm{Ca}^{2+}$ transport in Saccharomyces cerevisiae. J. Exp. Biol. 196: 157-166.

- 1994b. Calcineurin-dependent growth control in Saccharomyces cerevisiae mutants lacking PMC1, a homolog of plasma membrane $\mathrm{Ca}^{2+}$ ATPases. J. Cell Biol. 124: 351-363.

1996. Calcineurin inhibits VCX1-dependent $\mathrm{H}^{+} / \mathrm{Ca}^{2+}$ exchange and induces $\mathrm{Ca}^{2+}$ ATPases in yeast. Mol. Cell. Biol. 16: 2226-2237.

Cyert, M.S. and Thorner, J. 1992. Regulatory subunit |CNB1 gene product) of yeast $\mathrm{Ca}^{2+} /$ calmodulin-dependent phosphoprotein phosphatases is required for adaptation to pheromone. Mol. Cell. Biol. 12: 3460-3469.

Cyert, M.S., Kunisawa, R., Kaim, D., and Thorner, J. 1991. Yeast has homologs (CNA1 and CNA2 gene products) of mammalian calcineurin, a calmodulin-regulated phosphoprotein phosphatase. Proc. Natl. Acad. Sci. 88: 7376-7380.

Epstein, C.J. 1995. Down Syndrome (Trisomy 21) (ed. C.R. Scriber, A.L. Beaudet, W.S. Sly, and D. Vaile), pp. McGrawHill, Inc.

Fruman, D.A., Pai, S.Y., Klee, C.B., Burakoff, S.J., and Bierer, B.E. 1996. Measurement of calcineurin phosphatase activity in cell extracts. Methods 9: 146-154.

Fuentes, J.J., Genescà, L., Kingsbury, T.J., Cunningham, K.W., Pérez-Riba, M., Estivill, X., and de la Luna, S. 2000. DSCR1, overexpressed in Down syndrome, is an inhibitor of calcineurin-mediated signaling pathways. Hum. Mol. Gen. (in press).

Fuentes, J.J., Pritchard, M.A., Planas, A.M., Bosch, A., Ferrer, I., and Estivill, X. 1995. A new human gene from the Down syndrome critical region encodes a proline-rich protein highly expressed in fetal brain and heart. Hum. Mol. Genet. 4: 1935-1944.

Geiser, J.R., van Tuinen, D., Brockerhoff, S.E., Neff, M.M., and Davis, T.N. 1991. Can calmodulin function without binding calcium? Cell 65: 949-959.

Hemenway, C.S. and Heitman, J. 1999. Calcineurin: Structure, 
function, and inhibition. Cell Biochem. Biophys. 30: 115-151.

Hubbard, M.J. and Cohen, P. 1993. On target with a new mechanism for the regulation of protein phosphorylation. Trends Biochem. Sci. 18: 172-177.

Hughes, S.M. 1998. Muscle development: Electrical control of gene expression. Curr. Biol. 8: 892-894.

Kashishian, A., Howard, M., Loh, C., Gallatin, W.M., Hoekstra, M.F., and Lai, Y. 1998. AKAP79 inhibits calcineurin through a site distinct from the immunophilin-binding region. J. Biol. Chem. 273: 27412-27419.

Klee, C.B., Ren, H., and Wang, X. 1998. Regulation of the calmodulin-stimulated protein phosphatase, calcineurin. J. Biol. Chem. 273: 13367-13370.

Krebs, J. 1998. The role of calcium in apoptosis. Biometals 11: 375-382.

Kuno, T., Tanaka, H., Mukai, H., Chang, C.D., Hiraga, K., Miyakawa, T., and Tanaka, C. 1991. cDNA cloning of a calcineurin B homolog in Saccharomyces cerevisiae. Biochem. Biophys. Res. Commun. 180: 1159-1163.

Lai, M.M., Burnett, P.E., Wolosker, H., Blackshaw, S., and Snyder, S.H. 1998. Cain, a novel physiologic protein inhibitor of calcineurin. J. Biol. Chem. 273: 18325-18331.

Leahy, K.P., Davies, K.J., Dull, M., Kort, J.J., Lawrence, K.W., and Crawford, D.R. 1999. adapt78, a stress-inducible mRNA, is related to the glucose-regulated protein family of genes. Arch. Biochem. Biophys. 368: 67-74.

Liu, J., Farmer, Jr., J., Lane, W.S., Friedman, J., Weissman, I., and Schreiber, S.L. 1991a. Calcineurin is a common target of cyclophilin-cyclosporin A and FKBP-FK506 complexes. Cell 66: $807-815$

Liu, J., Albers, M.W., Wandless, T.J., Luan, S., Alberg, D.G., Belshaw, P.J., Cohen, P., MacKintosh, C., Klee, C.B., and Schreiber, S.L. 1992. Inhibition of T-cell signaling by immunophilin-ligand complexes correlates with loss of calcineurin phosphatase activity. Biochemistry 31: 3896-3901.

Liu, Y., Ishii, S., Tokai, M., Tsutsumi, H., Ohki, O., Akada, R., Tanaka, K., Tsuchiya, E., Fukui, S., and Miyakawa, T. 1991b. The Saccharomyces cerevisiae genes (CMP1 and CMP2) encoding calmodulin-binding proteins homologous to the catalytic subunit of mammalian protein phosphatase 2B. Mol. Gen. Genet. 227: 52-59.

Mansuy, I.M., Mayford, M., Jacob, B., Kandel, E.R., and Bach, M.E. 1998. Restricted and regulated overexpression reveals calcineurin as a key component in the transition from shortterm to long-term memory. Cell 92: 39-49.

Matheos, D.P., Kingsbury, T.J., Ahsan, U.S., and Cunningham, K.W. 1997. Tcn1p/Crzlp, a calcineurin-dependent transcription factor that differentially regulates gene expression in Saccharomyces cerevisiae. Genes \& Dev. 11: 3445-3458.

Mendizabal, I., Rios, G., Mulet, J.M., Serrano, R., and de Larrinoa, I.F. 1998. Yeast putative transcription factors involved in salt tolerance. FEBS Lett. 425: 323-328.

Miseta, A., Kellermayer, R., Aiello, D.P., Fu, L., and Bedwell, D.M. 1999. The vacuolar $\mathrm{Ca}^{2+} / \mathrm{H}^{+}$exchanger Vcxlp/Humlp tightly controls cytosolic $\mathrm{Ca}^{2+}$ levels in $S$. cerevisiae. FEBS Lett. 451: 132-136.

Miyazaki, T., Kanou, Y., Murata, Y., Ohmori, S., Niwa, T., Maeda, K., Yamamura, H., and Seo, H. 1996. Molecular cloning of a novel thyroid hormone-responsive gene, ZAKI-4, in human skin fibroblasts. J. Biol. Chem. 271: 14567-14571.

Molkentin, J.D., Lu, J.R., Antos, C.L., Markham, B., Richardson, J., Robbins, J., Grant, S.R., and Olson, E.N. 1998. A calcineurin-dependent transcriptional pathway for cardiac hypertrophy. Cell 93: 215-228.

Moser, M.J., Geiser, J.R., and Davis, T.N. 1996. $\mathrm{Ca}^{2+}$-calmodulin promotes survival of pheromone-induced growth arrest by activation of calcineurin and $\mathrm{Ca}^{2+}$-calmodulin-dependent protein kinase. Mol. Cell. Biol. 16: 4824-4831.

Pozos, T.C., Sekler, I., and Cyert, M.S. 1996. The product of HUM1, a novel yeast gene, is required for vacuolar $\mathrm{Ca}^{2+} / \mathrm{H}^{+}$ exchange and is related to mammalian $\mathrm{Na}^{+} / \mathrm{Ca}^{2+}$ exchangers. Mol. Cell. Biol. 16: 3730-3741.

Rao, A., Luo, C., and Hogan, P.G. 1997. Transcription factors of the NFAT family: Regulation and function. Annu. Rev. Immunol. 15: 707-747.

Rothermel, B., Vega, R.B., Yang, J., Wu, H., Bassel-Duby, R., and Williams, R.S. 2000. A protein encoded within the Down syndrome critical region is enriched in striated muscles and inhibits calcineurin signaling. I. Biol. Chem. 275: 87198725.

Sagoo, J.K., Fruman, D.A., Wesselborg, S., Walsh, C.T., and Bierer, B.E. 1996. Competitive inhibition of calcineurin phosphatase activity by its autoinhibitory domain. Biochem. J. 320: 879-884.

Shibasaki, F. and McKeon, F. 1995. Calcineurin functions in $\mathrm{Ca}^{2+}$-activated cell death in mammalian cells. J. Cell. Biol. 131: 735-743.

Sikorski, R.S. and Hieter, P. 1989. A system of shuttle vectors and yeast host strains designed for efficient manipulation of DNA in Saccharomyces cerevisiae. Genetics 122: 19-27.

Sim, A.T. and Scott, J.D. 1999. Targeting of PKA, PKC, and protein phosphatases to cellular microdomains. Cell. Calcium 26: 209-217.

Stathopoulos, A.M. and Cyert, M.S. 1997. Calcineurin acts through the CRZ1/TCN1 encoded transcription factor to regulate gene expression in yeast. Genes \& Dev. 11:34323444.

Stathopoulos-Gerontides, A., Guo, J.J., and Cyert, M.S. 1999. Yeast calcineurin regulates nuclear localization of the Crzlp transcription factor through dephosphorylation. Genes \& Dev. 13: 798-803.

Sun, L., Youn, H.D., Loh, C., Stolow, M., He, W., and Liu, J.O. 1998. Cabin 1, a negative regulator for calcineurin signaling in T lymphocytes. Immunity 8: 703-711.

Sussman, M.A., Lim, H.W., Gude, N., Taigen, T., Olson, E.N., Robbins, J., Colbert, M.C., Gualberto, A., Wieczorek, D.F., and Molkentin, J.D. 1998. Prevention of cardiac hypertrophy in mice by calcineurin inhibition. Science 281: 1690-1693.

Tung, H.Y., Wang, W., and Chan, C.S. 1995. Regulation of chromosome segregation by Glc8p, a structural homolog of mammalian inhibitor 2 that functions as both an activator and an inhibitor of yeast protein phosphatase 1. Mol. Cell. Biol. 15: 6064-6074.

Wang, X., Culotta, V.C., and B., K.C. 1996. Superoxide dismutase protects calcineurin from inactivation. Nature 383: 434-437.

Winder, D.G., Mansuy, I.M., Osman, M., Moallem, T.M., and Kandel, E.R. 1998. Genetic and pharmacological evidence for a novel, intermediate phase of long-term potentiation suppressed by calcineurin. Cell 92: 25-37.

Ye, R.R. and Bretscher, A. 1992. Identification and molecular characterization of the calmodulin-binding subunit gene (CMP1) of protein phosphatase 2B from Saccharomyces cerevisiae. An alpha-factor inducible gene. Eur. J. Biochem. 204: 713-723.

Youn, H.D., Sun, L., Prywes, R., and Liu, J.O. 1999. Apoptosis of $\mathrm{T}$ cells mediated by $\mathrm{Ca}^{2+}$-induced release of the transcription factor MEF2. Science 286: 790-793.

Zhu, J., Shibasaki, F., Price, R., Guillemot, J.C., Yano, T., Dotsch, V., Wagner, G., Ferrara, P., and McKeon, F. 1998. Intramolecular masking of nuclear import signal on NF-AT4 by casein kinase I and MEKK1. Cell 93: 851-861. 


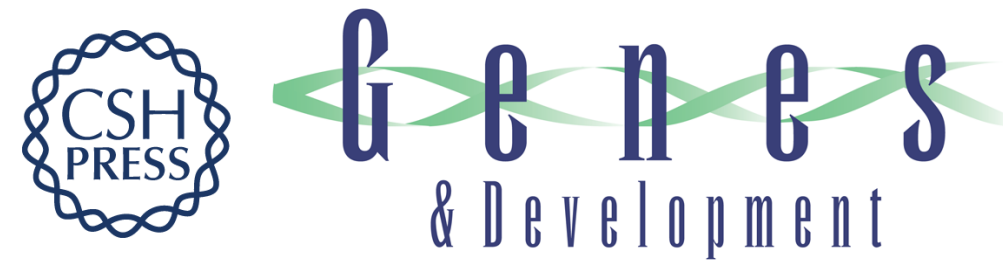

\section{A conserved family of calcineurin regulators}

Tami J. Kingsbury and Kyle W. Cunningham

Genes Dev. 2000, 14:

Access the most recent version at doi:10.1101/gad.14.13.1595

References This article cites 49 articles, 24 of which can be accessed free at: http://genesdev.cshlp.org/content/14/13/1595.full.html\#ref-list-1

License

Email Alerting Receive free email alerts when new articles cite this article - sign up in the box at the top Service right corner of the article or click here.

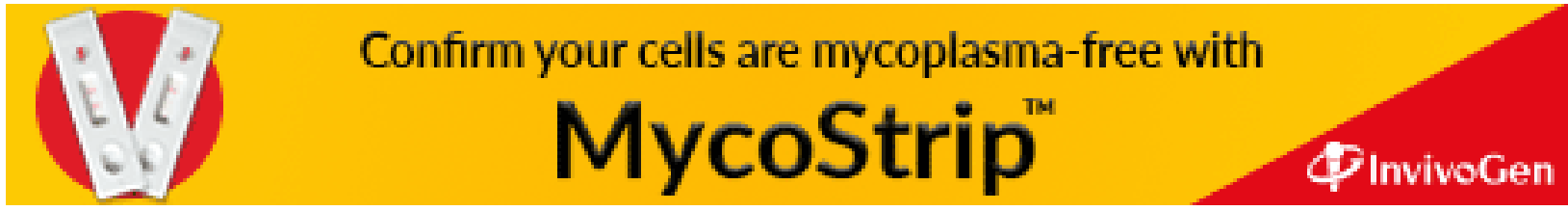

